\title{
Sentence to Document Level Emotion Tagging - A Coarse-Grained Study on Bengali Blogs
}

\author{
Dipankar Das and Sivaji Bandyopadhyay \\ Department of Computer Science and Engineering, Jadavpur University, India \\ dipankar.dipni12005@gmail.com, sivaji_cse_ju@yahoo.com
}

\begin{abstract}
This paper presents the identification of document level emotions from the sentential emotions obtained at word level granularity. Each of the Bengali blog documents consists of a topic and corresponding user comments. Sense weight based average scoring technique for assigning sentential emotion tag follows the word level emotion tagging using Support Vector Machine (SVM) approach. Cumulative summation of sentential emotion scores is assigned to each document considering the combinations of some heuristic features. An average F-Score of $59.32 \%$ with respect to all emotion classes is achieved on 95 documents on the development set by incorporating the best feature combination into account. Instead of assigning a single emotion tag to a document, each document is assigned with the best two emotion tags according to the ordered emotion scores obtained. The best two system assigned emotion tags of each document are compared against best two human annotated emotion tags. Evaluation of 110 test documents yields an average F-Score of 59.50\% with respect to all emotion classes.
\end{abstract}

Keywords: Document, SVM, Emotion Tagging, Heuristic Features.

\section{Introduction}

From the classification of reviews [18] or newspaper articles [15] to Question Answering systems [6] and modern Information Retrieval systems [4], all the areas are increasingly incorporating emotion analysis within their scope. It sometimes important to track users' emotion expressed in online forums or blogs or twitters for different applications such as sentiment review, customer management, stock exchange prediction etc. Blogs are the communicative and informative repository of text based emotional contents in the Web 2.0 [15]. Researches on emotion show that blogs play the role of a substrate to analyze the reactions of different emotional enzymes. In general, sentence level emotion identification plays an important role to track emotions or to find out the cues for generating such emotions or to properly identify it. Sentences as the information units of any document identify the overall document level emotion whereas emotion of individual sentence in the documents is based on the emotions expressed by the word(s) $[3,16]$.

In the present task, each of the Bengali blog documents containing individual topic and corresponding user comments is collected from the web blog archive 
(www.amarblog.com). Each of the blog documents is annotated with Ekman's (1993) [17] six basic emotion tags. The method adopted for assigning sentential emotion tag is based on word level constituents follows the same approach as in [9]. But, instead of using Conditional Random Field (CRF) [2] based classifier, Support Vector Machine (SVM) [5] is used for word level emotion tagging in the present task. It is observed that SVM outperforms CRF in word level emotion tagging. The sense weight based average scoring technique [9] is applied for assigning sentential emotion tags based on word level emotion tagged constituents. The document level emotion tagging with emotion scores from emotion tagged sentences is carried out based on some combinations of heuristic features (e.g. emotion tag of the title sentence or end sentence of a topic, emotion tags assigned to an overall topic, most frequent emotion tags expressed in user comment portions of a document, identical emotions that appear in the longest series of tagged sentences etc.). The best two emotion tags are assigned to a document based on the ordered maximum emotion scores obtained. The development set gives the best average $F$-Score of $59.32 \%$ after applying all possible feature combinations. Evaluation is carried out against the best two annotated emotion tags of 110 test documents containing 1298 user comments sections. Average F-Score of $59.50 \%$ has been achieved with respect to all emotion classes.

The rest of the paper is organized as follows. Section 2 describes the related work done in this area. Corpus preparation followed by sentence level emotion tagging is discussed in Section 3. Document level emotion tagging is mentioned in Section 4. Section 5 describes subsequent emotion class wise evaluation with respect to the best two emotion tags. Finally Section 6 concludes the paper.

\section{Related Work}

Several efforts have been attempted by the natural language processing researchers to identify emotion at different level of granularities. In [16], work on opinions mining at word, sentence and document levels from news and web blog articles has been conducted along with opinion summarization. The present work is similar only in the sense that both of the works adopted the granular fashion to represent and solve the problem related to opinion or emotion. But tour approach differs in assigning document level emotion tags along with the knowledge of heuristic features.

In [13, 14], Support Vector Machine (SVM) based classifier was used on the blog data to classify the documents according to the mood of the author during writing. The authors used emoticons in LiveJournal posts to train a mood classifier at the document level. Another related article described in [15] has focused on the classification of news articles into the readers' emotions instead of the authors'. The work discussed in [8] used Yahoo! Kimo Blog as corpora to build emotion lexicons. In their studies, emoticons are used to identify emotions associated with textual keywords. In the present task, the perspective of reader is selected only to judge the emotional counterpart. The emoticons of Bengali blog documents are also considered in the present task.

The task described in [12] adopted different unsupervised, supervised and semi supervised strategies to identify and classify emotions. The researchers of [8] have done an experiment of the emotion classification task on web blog corpora using 
SVM and CRF machine learning techniques. It is observed that the CRF classifiers outperform SVM classifiers in case of document level emotion detection. In contrast, the present task shows that SVM outperforms over CRF in word level emotion detection as CRF suffers from sequence labeling issues used for tagging emotion to discrete word tokens. However, most of the related work has been conducted for English. Bengali is less privileged and less computerized than English. Works on emotion analysis in Bengali have started recently $[9,10]$. The present work differs in the respect that the knowledge extracted from different heuristic features and their combinations play a novel role in document level emotion tagging.

\section{Word to Sentence Level Emotion Tagging}

\subsection{Corpus Preparation}

As Bengali is very resource constraint language, the emotion annotated Bengali blog corpus [9] is being developed manually. A small portion has been considered in the present task. The blog documents stored in the format as shown in Figure 1 are retrieved from the web blog archive (www.amarblog.com). Each of the blog documents is assigned with a unique identifier (docid) followed by a section devoted for topic section and several sections devoted for different users' comments. Each of the sections of users' comment that is associated with corresponding user id (uid) may also contain the comments of other users. Such overlapped comments are differentiated using their corresponding user ids (uid).

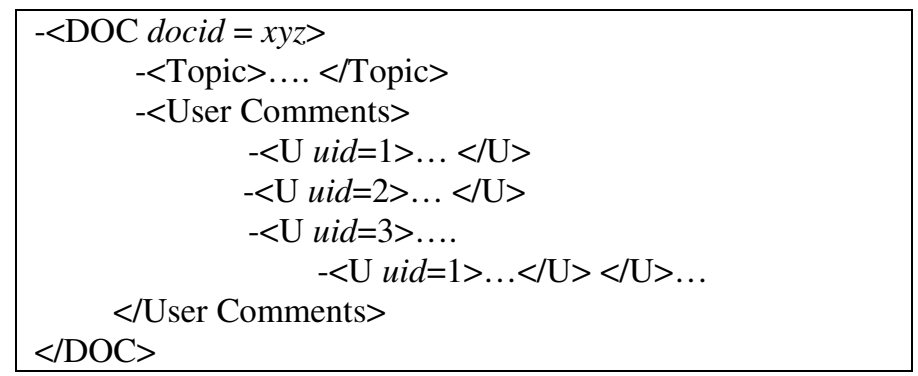

Fig. 1. General structure of a blog document

Total of 24678 sentences are employed in the present task. Although we have considered 95 and 110 documents as our development and test sets respectively but 300 and 200 development and test sentences of [9] are used in the present task for conducting different experiments and comparative evaluations of sentential emotion tagging. Out of total 1200 sentences collected from 14 different comic related blog documents, rest 700 sentences are considered for SVM based training of the word level emotion tagging system. 


\subsection{Sentential Emotion Tagging}

In the present task, the technique adopted for assigning sentential emotion tags is similar with the approach that was considered in [9]. But, instead of using Conditional Random Field (CRF), the Support Vector Machine (SVM) based classifier is used in the present task to classify each word into any of the Ekman's(1993) six emotion categories. Feature plays a crucial rule in any machine-learning framework. Hence, among 10 active singleton features of [9], 9 features have been employed to accomplish the current task. Instead of SentiWordNet emotion word, the Bengali WordNet Affect [11] has been used in this task to identify feature for emotion words. Different unigram and bi-gram context features (word level as well as POS tag level) have been applied. The features are as follows.

- POS information (adjective, verb, noun, adverb)

- First sentence in a topic or title sentence [7]

- Emotion words of Bengali WordNet Affect (e.g. क्্যাा (khyama) [pardon] )

- Reduplication (e.g., bhallo bhallo [good good], khokhono khokhono [when when] etc.)

- Question words (ki [what], keno [why] etc.)

- Colloquial / Foreign words (e.g., kshyama [pardon] etc.) and foreign words (e.g. Thanks, gossya [anger] etc.)

- $\quad$ Special punctuation symbols (!,@,?..)

- Quoted sentence ("you are 2 good man")

- Sentence Length $(>=8,<15)$

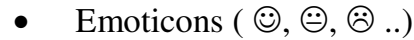

The CRF classifier generally performs the classification task for sequence labeling problem, and thus it carries out word level classification task with a significant loss of word level emotional constituents. As SVM gives better performance in discrete (e.g. word) information tagging, the improvement is found in the word level emotion classification task. The comparative results of CRF and SVM for word level emotion tagging are shown in Table 1. The improvement of word level emotion tagging system also carries its effects to the sentential emotion tagging.

Table 1. Word level emotion tagging accuracies (\%) for six emotion classes on the Development and Test set using CRF and SVM

\begin{tabular}{lccc}
\hline Emotion Classes & Development Set (\# words) & \multicolumn{2}{c}{ Test Set (\#words) } \\
& & CRF-based model & SVM-based model \\
\hline Happy & $61.17(185)$ & $67.67(106)$ & $69.55(106)$ \\
Sad & $64.63(151)$ & $63.12(143)$ & $65.34(143)$ \\
Anger & $62.85(136)$ & $51.00(70)$ & $56.15(70)$ \\
Disgust & $68.66(100)$ & $49.75(65)$ & $53.35(65)$ \\
Fear & $63.38(96)$ & $52.46(37)$ & $54.78(37)$ \\
Surprise & $79.05(230)$ & $68.23(204)$ & $69.37(204)$ \\
\hline
\end{tabular}

The default emotion tag weights for six emotion types are taken from $[9,10]$. In the method, the basic six words "happy", "sad", "anger", "disgust", "fear" and "surprise" are chosen as the seed words corresponding to each emotion type. The 
positive and negative scores in the English SentiWordNet [1] for each synset in which each of these seed words appear are retrieved and the average of the scores is fixed as the Sense_Tag_Weight (STW) of that particular emotion tag.

The present work differs from the approach in [9] while assigning emotion scores and sentential emotion tags to the blog sentences. In the present method, the emotion tagged words have been considered instead of depending on the fixed emotion tag weights assigned for the words. For supporting the task, a Bengali SentiWordNet is being developed by replacing each word entry in the synonymous set of the English SentiWordNet by its possible set of Bengali synsets using a synset based English to Bengali bilingual dictionary being developed as part of the EILMT ${ }^{1}$ project.

Each word tagged with a particular emotion type is searched in the Bengali SentiWordNet and the positive and negative scores of the word are retrieved from the SentiWordNet. The average of the scores is fixed as the Sense_Tag_Weight (STW) for the emotion tag assigned to the word. If an emotion tagged word is not found in the Bengali SentiWordNet, the default weight calculated earlier is assigned for that word. The total Sense_Tag_Weight (STW) for each emotion tag $i$ is calculated by summing up the STWs of all assigned emotion tags with type $i$. Stemming is included during the searching process. Bengali, like any other Indian languages, is morphologically very rich. Different suffixes may be attached to a word depending on the various features (e.g. the features for a Bengali verb are Tense, Aspect, and Person). A Bengali stemmer uses a suffix list to identify the stem form of the word.

Apart from the search technique, the sentential emotion tagging is carried out in a similar fashion that was adopted by [9]. Each sentence is assigned with a Sense_Weight_Score (SWS) for each emotion type. The weight is calculated by dividing the total STW of all occurrences of that emotion tag in the sentence by the total STW of all types of emotion tags present in that sentence. The sentence is assigned with the emotion tags for which the sentence level Sense_Weight_Score (SWS) is highest. The sentences are tagged as neutral type if for all emotion tags, the total Sense_Weight_Scores (SWS) produce zero (0) emotion score. The post processing strategies [9] related to negative words have been incorporated in the present system. The comparative results of the CRF based model with SVM for sentence level emotion tagging is shown in Table 2.

Table 2. CRF and SVM based test set accuracies (in \%) per emotion class for sentential emotion tagging

\begin{tabular}{cccc}
\hline $\begin{array}{c}\text { Emotion Class (Total } \\
\text { \# sentences) }\end{array}$ & CRF-based model & SVM- based model \\
\hline \multicolumn{2}{c}{ happy $\quad(40)$} & 65.28 & 66.05 \\
sad & $(41)$ & 66.42 & 68.12 \\
angry & $(32)$ & 60.28 & 62.77 \\
disgust & $(21)$ & 52.18 & 53.54 \\
fear & $(23)$ & 57.14 & 60.11 \\
surprise & $(43)$ & 66.45 & 69.82 \\
\hline
\end{tabular}

\footnotetext{
${ }^{1}$ English to Indian Languages Machine Translation (EILMT) is a TDIL project undertaken by the consortium of different premier institutes and sponsored by MCIT, Govt. of India.
} 


\section{Document Level Emotion Tagging}

Assigning a single emotion tag to a particular document does not always bear the actual emotions present in that document. This module identifies document level emotion tags and their associated weights based on the sentence level emotion tags along with the contribution of some heuristic feature combinations. Emotion_Weight_Score (EWS) based technique applied on the sentence level emotion tags produces six possible emotion scores for a document. But, the evaluation is carried out for the best two emotion tags only.

\subsection{Calculation of Document Level Emotion Tag Weights}

Each document is assigned with an Emotion_Weight_Score (EWS) for each of the six emotion tags. In general, the document level Emotion_Weight_Score (EWS) for a particular emotion tag is calculated by summing up the total Sense_Weight_Scores (SWS) of all occurrences of the sentential tags corresponding to that emotion category, i.e., EWS $i=\sum \mathrm{SWS} i$, where SWS $i$ is the sentence level Sense_Weight_Score (SWS) for the emotion tag $i$ in the document. Each document is assigned with two document emotion tags DET $i$ and DET $j$, for which EWS $i$ is the highest and EWS $j$ is the second highest Emotion_Weight_Score. SET $i=[\mathrm{Max} i=1$ to $6(\mathrm{EWS} i)]$ and SET $j=[\mathrm{Max} j=1$ to $6 \& \& j \neq i(\mathrm{EWS} j)]$. But, the document level emotion tagging incorporates the heuristic features and their combinations into consideration.

\subsection{Heuristic Features}

Document level emotion identification depends not only on the emotion expressed in the sentential constituents but also on the combination of different characteristic features of that document (e.g. the blog structure). Irrespective of linguistic attributes alone, blog itself contains some special inherent features that help in identifying emotion at sentence as well as document level. In the present task, the following 7 active features identified heuristically for the document level emotion tagging.

(I) Emotion tags of the title sentence $(95,110)$

(II) Emotion tags of the end sentence of a topic $(95,110)$

(III) Emotion tags assigned to an overall topic $(95,110)$

(IV) Emotion tags for user comment portions of a document $(1156,1298)$

(V) Most frequent emotion tags identified from the document $(95,110)$

(VI) Identical emotions that appear in the longest series of tagged sentences $(67,61)$ (Yang et al., 2007)

(VII) Emotion tags of the largest section among all of the user comments' sections $(1274,1322)$

The numeric figures in brackets after each heuristic feature denote the number of times the corresponding feature has appeared in the development and test set respectively. Each of the documents contains a title and a topic and hence the frequencies of the first three features are same as the number of documents. The development and test documents contain a total of 1156 and 1298 number of sections specified for user comments only. The emotions reflected inside user comment 
sections are helpful for predicting the emotions at document level. The emphasis is also given to the frequency of the emotion tags identified at document level. Sometimes comments portion of a user contain other user comments in a nested fashion. Hence, one or more comment sections contain a large number of emotional sentences than other sections. This feature enhances the continuity of emotion in document for predicting the overall emotions expressed in the document. The contributions of the features alone and in combination with other features have been evaluated on 95 developmental documents. Information related to the frequency of different features is shown in Table 3 on basis of importance. It has been observed that the emotion classes e.g. fear, disgust contain less frequent information regarding features I, II and V.

Table 3. Frequencies of seven features per emotion class in the development and test sets respectively

\begin{tabular}{ccc}
\hline $\begin{array}{c}\text { Emotion } \\
\text { Classes }\end{array}$ & \multicolumn{3}{c}{ Information (Feature id, \#Frequency) } \\
Test Set
\end{tabular}

\section{Evaluation}

Emotion tags are assigned to each document by counting the total number of sentential emotion tags and summing up the associated average weight scores. The best two tags are assigned to the document based on the maximum and next to maximum emotion scores obtained. The F-score value is calculated on the development set for each of the features and individual contribution of each feature is measured. It is found that the contribution of each feature is not uniform and can be fairly distinguished according to the level of importance. For example, the combination of topic as well as user comments is identified as a contributory feature pair denoted by the experiment id $i i(8)$. The contributions of all the features are not mentioned but the detailed experimental evaluation and associated results regarding features are shown in Table 4. The emotion tags corresponding to maximum and next to maximum Emotion_Weight_Scores (EWS) of a document are considered as the probable candidate emotion tags. The set, namely, GSDT (Gold Standard Document Tag) contains two emotion tags assigned to a document in the gold standard annotated corpus and is defined as $\{d \max 1, d \max 2\}$. Document level emotion tagging module 
has generated the set SGDT (System Generated Document Tag) that contains two probable candidate emotion tags to a document based on their ordered Emotion_Weight_Scores (EWS) and the set is described as $\left\{d \max 1^{\prime}, d \max 2^{\prime}\right\}$. The emotion tags dmaxland dmax 2 correspond to the maximum and next to maximum scores for two emotion tags assigned to a document in the gold standard corpus and $d \max 1^{\prime}$ and $d \max _{2}$ ' are the maximum and next to maximum scores for two emotion tags to a document generated by the system.

Table 4. F-scores (in \%) of different heuristic features and their combinations on development set

\begin{tabular}{|c|c|c|}
\hline Expt. Id. & Current Feature Set & F-Score (in \%) \\
\hline \multirow[t]{7}{*}{$\overline{\mathbf{i}}$} & (1) Emotion tags of the title sentence & 31.12 \\
\hline & (2) Emotion tags of the end sentence of a topic & 28.25 \\
\hline & (3) Emotion tags assigned to an overall topic & 48.87 \\
\hline & $\begin{array}{l}\text { (4) Emotion tags for user comment portions of a } \\
\text { document }\end{array}$ & 52.66 \\
\hline & $\begin{array}{l}\text { (5) Most frequent emotion tags identified from the } \\
\text { document }\end{array}$ & 53.95 \\
\hline & $\begin{array}{l}\text { (6) Identical emotions that appear in the longest } \\
\text { series of tagged sentences }\end{array}$ & 37.29 \\
\hline & $\begin{array}{l}\text { (7) Emotion tags of the largest section among all of } \\
\text { the user comments' sections. }\end{array}$ & 35.11 \\
\hline \multirow[t]{7}{*}{ ii } & (8). $\quad \mathrm{i}(3)+\mathrm{i}(4)$ & 57.32 \\
\hline & (9). $\quad \mathrm{i}(3)+\mathrm{i}(5)$ & 56.55 \\
\hline & (10). $\mathrm{i}(3)+\mathrm{i}(7)$ & 55.42 \\
\hline & (11). $\mathrm{i}(4)+\mathrm{i}(5)$ & 54.87 \\
\hline & $(12) . i(4)+i(6)$ & 53.25 \\
\hline & (13). i(4)+i(7) & 55.57 \\
\hline & (14). $\mathrm{ii}(8)+\mathrm{i}(6)$ & 58.54 \\
\hline \multirow[t]{4}{*}{ iii } & (15). $\mathrm{ii}(8)+\mathrm{i}(7)$ & 58.04 \\
\hline & (16). ii(11)+i(6) & 56.21 \\
\hline & (17). $\mathrm{ii}(11)+\mathrm{i}(7)$ & 56.55 \\
\hline & (18). iii(14)+i(5) & 59.32 \\
\hline \multirow{2}{*}{ iv } & (19). iii(13)+i(5) & 58.70 \\
\hline & (20). $\mathrm{iii}(15)+\mathrm{iii}(16)$ & 58.02 \\
\hline
\end{tabular}

The $F$-Score for each emotion tag pair is measured by considering the number of system generated document tags that are matched correctly with annotated tags. The final average $F$-Score is calculated for each emotion class considering any four combinations of the two sets. The tagged documents are evaluated against the manually annotated 95 gold standard documents of the development set and then applied on 110 test documents. It is observed that $59.32 \%$ F-score has been achieved with these four combinations on a development set. The corresponding feature combination that gives best $F$-Score on the development set is applied for 110 test documents and finally an average $F$-Score of $59.50 \%$ is achieved. It is also observed that the emotions expressed in the title of the document do not convey the actual emotions expresses inside the document. Hence, the coarse grained evaluation based 
on the heuristic features helps to predict the document level emotions extensively. An important observation is that, as the number of feature instances varies in the emotion classes, they have imposed an impact on the document level tagging for that emotion classes respectively. It has to be mentioned that the performance of the system in terms of $F$-Score has not improved significantly by conducting the extended evaluation with adding of more than two tags in GSDT and SGDT sets.

Table 5. Evaluation of Document Level Emotion Tagging

\begin{tabular}{|c|c|c|c|}
\hline \multirow{2}{*}{\multicolumn{2}{|c|}{$\begin{array}{c}\text { Tag Combination of GSDT and } \\
\text { SGDT }\end{array}$}} & \multicolumn{2}{|c|}{ Test Documents（\#110) } \\
\hline & & F-Score & Average F-Score \\
\hline \multirow{5}{*}{ happy } & & & \\
\hline & $\left\{d \max 1, d \max 2^{\prime}\right\}$ & 58.11 & \\
\hline & $\left\{d \max 2, d \max 1^{\prime}\right\}$ & 57.08 & 58.74 \\
\hline & $\left\{d \max 2, d \max 2^{\prime}\right\}$ & 58.56 & \\
\hline & $\left\{\mathrm{d} \max 1, \quad d \max 1^{\prime}\right\}$ & 60.98 & \\
\hline \multirow[t]{4}{*}{ sad } & $\left\{d \max 1, d \max 2^{\prime}\right\}$ & 61.08 & \\
\hline & $\left\{d \max 2, d \max 1^{\prime}\right\}$ & 59.77 & 60.78 \\
\hline & $\left\{d \max 2, d \max 2^{\prime}\right\}$ & 61.32 & \\
\hline & $\left\{\mathrm{d} \max 1, \quad d \max 1^{\prime}\right\}$ & 61.57 & \\
\hline \multirow[t]{4}{*}{ angry } & $\{d \max 1, d \max 2\}$ & 59.22 & \\
\hline & $\left\{d \max 2, d \max 1^{\prime}\right\}$ & 59.69 & 60.25 \\
\hline & $\{d \max 2, d \max 2\}$ & 60.54 & \\
\hline & $\left\{\mathrm{d} \max 1, \quad d \max 1^{\prime}\right\}$ & 57.87 & \\
\hline \multirow[t]{4}{*}{ disgust } & $\left\{d \max 1, d \max 2^{\prime}\right\}$ & 58.06 & \\
\hline & $\left\{d \max 2, d \max 1^{\prime}\right\}$ & 58.17 & 58.40 \\
\hline & $\left\{d \max 2, d \max 2^{\prime}\right\}$ & 59.51 & \\
\hline & $\left\{\mathrm{d} \max 1, \quad d \max 1^{\prime}\right\}$ & 57.34 & \\
\hline \multirow[t]{4}{*}{ fear } & $\{d \max 1, d \max 2\}$ & 57.81 & \\
\hline & $\left\{d \max 2, d \max l^{\prime}\right\}$ & 59.37 & 58.19 \\
\hline & $\{d \max 2, d \max 2\}$ & 58.25 & \\
\hline & $\left\{\mathrm{d} \max 1, \quad d \max 1^{\prime}\right\}$ & 60.33 & \\
\hline \multirow[t]{3}{*}{ surprise } & $\left\{d \max 1, d \max 2^{\prime}\right\}$ & 60.81 & \\
\hline & $\left\{d \max 2, d \max l^{\prime}\right\}$ & 61.37 & 60.69 \\
\hline & $\left\{d \max 2, d \max 2^{\prime}\right\}$ & 60.25 & \\
\hline
\end{tabular}

\section{Conclusion}

In the present task, the sentence level emotion tags are used to assign document level emotion tags. The resulting document level emotion tagger can be used in an emotion based information retrieval system where retrieved documents will match the user defined query word(s) and emotion specification. The idea of assigning two emotion tags to the documents can then be related to the ranking of the retrieved sentences and documents. Emotion analysis related to the effect of metaphors (especially in blogs) is the research area to be explored in future. But the clause level analysis of the complex emotional sentences may be an area for further studies. 


\section{References}

1. Esuli, A., Sebastiani, F.: SENTIWORDNET: A Publicly Available Lexical Resource for Opinion Mining. In: LREC-06 (2006)

2. McCallum, A., Pereira, F., Lafferty, J.: Conditional Random Fields: Probabilistic Models for Segmenting and labeling Sequence Data. ISBN, 282-289 (2001)

3. Vincent, B., Xu, L., Chesley, P., Srhari, R.K.: Using verbs and adjectives to automatically classify blog sentiment. In: Proceedings of AAAI-CAAW-06, the Spring Symposia (2006)

4. Pang, B., Lee, L.: Foundations and Trends in Information Retrieval 2, vol. 1-2, pp. 1-135 (2008)

5. Cortes, C., Vapnik, V.: Support-Vector Network. Machine Learning 20, 273-297 (1995)

6. Claire, C., Janyce, W., Theresa, W., Litman Diane, J.: Combining Low-Level and Summary Representations of Opinions for Multi-Perspective Question Answering. New Directions in Question Answering, 20-27 (2003)

7. Alm, C.O., Roth, D., Sproat, R.: Emotions from text: machine learning for text-based emotion prediction. In: Proceedings of the Conference on HLT (EMNP) Vancouver, British Columbia, Canada, pp. 579-586 (2005)

8. Yang, C., Lin, K.H.-Y., Chen, H.-H.: Emotion classification Using Web Blog Corpora. In: IEEE, WIC, ACM International Conference on Web Intelligence, pp. 275-278 (2007)

9. Das, D., Bandyopadhyay, S.: Emotion Tagging - A Comparative Study on Bengali and English Blogs. In: ICON-09, India, pp. 177-184 (2009)

10. Das, D., Bandyopadhyay, S.: Word to Sentence Level Emotion Tagging for Bengali Blogs. In: ACL-IJCNLP 2009, Suntec, Singapore, pp. 149-152 (2009)

11. Das, D., Bandyopadhyay, S.: Developing Bengali WordNet Affect for Analyzing Emotion. In: ICCPOL-2010, California, USA (2010)

12. Sebastiani, F.: Machine learning in automated text categorization. ACM Computing Surveys 34(1) (2002)

13. Mishne, G.: Experiments with Mood Classification in Blog Posts. In: Proceedings of 1st Workshop on Stylistic Analysis of Text for Information Access (2005)

14. Mishne, G., de Rijke, M.: Capturing Global Mood Levels using Blog Posts. In: Proceedings of AAAI, Spring Symposium on Computational Approaches to Analysing Weblogs, pp. 145-152 (2006)

15. Lin, K.H.-Y., Yang, C., Chen, H.-H.: What Emotions News Articles Trigger in Their Readers? In: Proceedings of SIGIR, pp. 733-734 (2007)

16. Ku, L.-W., Liang, Y.-T., Chen, H.-H.: Opinion extraction, summarization and tracking in news and blog corpora. In: AAAI-2006 Spring Symposium on Computational Approaches to Analyzing Weblogs, AAAI Technical Report, pp. 100-107 (2006)

17. Ekman, P.: Facial expression and emotion. American Psychologist 48(4), 384-392 (1993)

18. Turney, P.D.: Thumbs Up or Thumbs Down? Semantic Orientation Applied to Unsupervised Classification of Reviews. In: Proceedings of the 40th Annual Meeting of the Association for Computational Linguistics, pp. 417-424 (2002) 